\title{
3D Inkjet Printed Radio Frequency Inductors and Capacitors
}

\author{
Mohammad Vaseem, Garret McKerricher, Atif Shamim \\ King Abdullah University of Science and Technology, Electrical Engineering Program, CEMSE Division, Thuwal, Saudi Arabia \\ mohammad.vaseem@kaust.edu.sa, garret.mckerricher@kaust.edu.sa, atif.shamim@kaust.edu.sa
}

\begin{abstract}
Inkjet printing has emerged as an ideal method for the fabrication of low cost and efficient electronic systems. However, most of the printed designs at present utilize 2D inkjet printing of metallic inks on conventional substrates. In order to have fully printed RF components, the substrate must also be printed. 3D printing of polymers can be an ideal mechanism for printing substrates, however typically such materials cannot handle high sintering temperatures $\left(>150{ }^{\circ} \mathrm{C}\right)$ required for nanoparticles based metallic inks. In this work, an all-inkjet printed process is demonstrated that utilizes 3D inkjet printing of a UV-cured dielectric material in combination with the printing of a particle free conductive silver organo-complex (SOC) ink for realization of inductors and capacitors. The processing temperature does not exceed $80{ }^{\circ} \mathrm{C}$ and still state of the art conductivity of $1 \times 10^{7} \mathrm{~S} / \mathrm{m}$ is achieved. Both the conductive ink and dielectric have roughness values under $500 \mathrm{~nm}$. The inductor and capacitor exhibit quality factors of 8 and 20 respectively in the high MHz and GHz regime.
\end{abstract}

Keywords - Inkjet printing; 3D; Multijet; radio frequency; capacitor; inductor; Silver

\section{INTRODUCTION}

Inkjet printing is transitioning from solely a graphic arts medium into a useful fabrication tool. The ability to deposit multiple materials and the scalability of inkjet systems with thousands of nozzles makes it possible to realize large and complex parts. One of the major advancements in inkjet printing has been the use of UV-cured acrylic materials. Like acrylic paints, inks can be made with vibrant colour and immediately cured upon exposure with a low-cost UV flood lamp. A major benefit of UV-cured ink is that the droplet is pinned, which restricts ink spreading due to the polymerization reaction that is immediately induced by the UV exposure. This concept can be taken beyond graphic art to form fully printed 3D parts. While the technology is still in its infancy, commercial 3D inkjet printers are now available from Stratasys and 3D Systems. A major advantage of the inkjet process over other 3D techniques is that multiple materials can be deposited together. So far this has been restricted to the colorful UV cured acrylics and wax/gel like support materials. An exciting opportunity exists for 3D inkjet printing of electronics if a metal could be integrated within the process. To date there have been several reports of 3D printed RF electronics, where the metal has been deposited by sputtering, aerosol, plating solutions, and conductive pastes.[1-4] In advancement, an inkjet printed metal integration into the UV cure material creating a fully 3D inkjet technique is require for rapid prototyping and electronics fabrication. However, the major difficulty with integrating the metal is the thermal compatibility with the 3D material.

It is no surprise that there have been several reports of inkjet printed inductors and capacitors in literature [5-8] however, due to the sensitivity to metal conductivity, thickness, and roughness, high quality inkjet-printed RF components combining $3 \mathrm{D}$ printing remain elusive. A recent report by $\mathrm{Wu}$ et al. shows a novel concept of 3D printing, together with syringe injection of a silver paste at $70{ }^{\circ} \mathrm{C}$ to create $3 \mathrm{D}$ inductors and capacitors; however these pastes achieve low conductivity of $2.8 \times 10^{5} \mathrm{~S} / \mathrm{m}$.[9]

This work demonstrates two orders of magnitude higher conductivity $1 \times 10^{7} \mathrm{~S} / \mathrm{m}$ at a low temperature $\left(80^{0} \mathrm{C}\right)$ only using a low cost IR lamp with a fully $3 \mathrm{D}$ inkjet printing process. This is capable of creating higher quality components. The key to the fully inkjet printing process is the materials involved, both the SOC ink and the UV cure polymer. The multi-layers fabricated inductor and capacitor exhibit quality factors of 8 and 20 respectively in the RF regime. Using this fully printed multi-layer process, various high quality microwave components can be realized.

\section{FABRICATION PROCESS}

The steps for the fabrication process are shown in Fig 1.

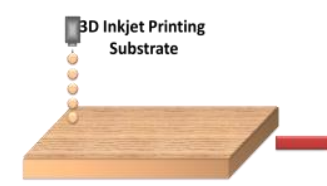

(i)

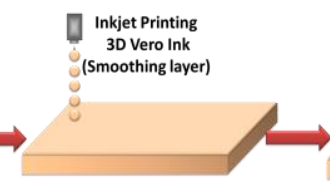

(ii)

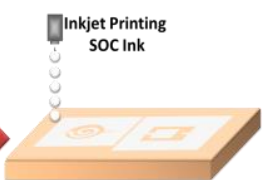

(iii) Infra-red (IR)
heating (5 min) (t)

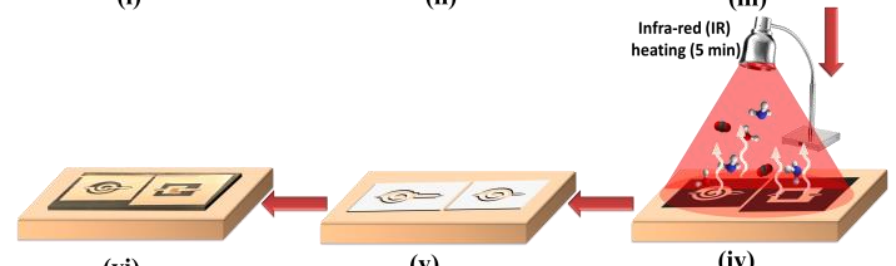

(vi)

(v)

(iv)

Fig. 1. Schematic presentation of fabrication process for multilayer 3D inkjet printed inductors and capacitors

First the UV cure material is printed with the Objet 3D printer allowing complex and thick shapes (step i). A challenge that 
still exists is the surface roughness of the printed material from the Objet printer. Surface roughness is especially detrimental in $\mathrm{RF}$ components where it results in additional loss in the devices. The white light interferometer measurement shows the wavy surface of printed material with $1.8 \mu \mathrm{m}$ RMS roughness. This was improved with the jetting of an additional layer of Vero ink with the Dimatix printer (step ii). After deposition, the ink is allowed to spread before UV curing and smooths the surface to $0.4 \mu \mathrm{m}$ RMS roughness. Successively, SOC ink is printed and cured with IR-heating (steps iii-iv). After IR curing conductive films are shown in (step v). They can now be integrated to create multilayers fully $3 \mathrm{D}$ inkjet printed $\mathrm{RF}$ components. The final module is shown in (step vi). Fig. 2 (a) is showing cross-sectional multilayers depiction for inductors and capacitors. Both the SOC and UV cure polymer have excellent jetting properties from the Dimatix printer and can be processed at $80{ }^{0} \mathrm{C}$. The final printed (b) inductor and (c) capacitor is showing in Fig. 2

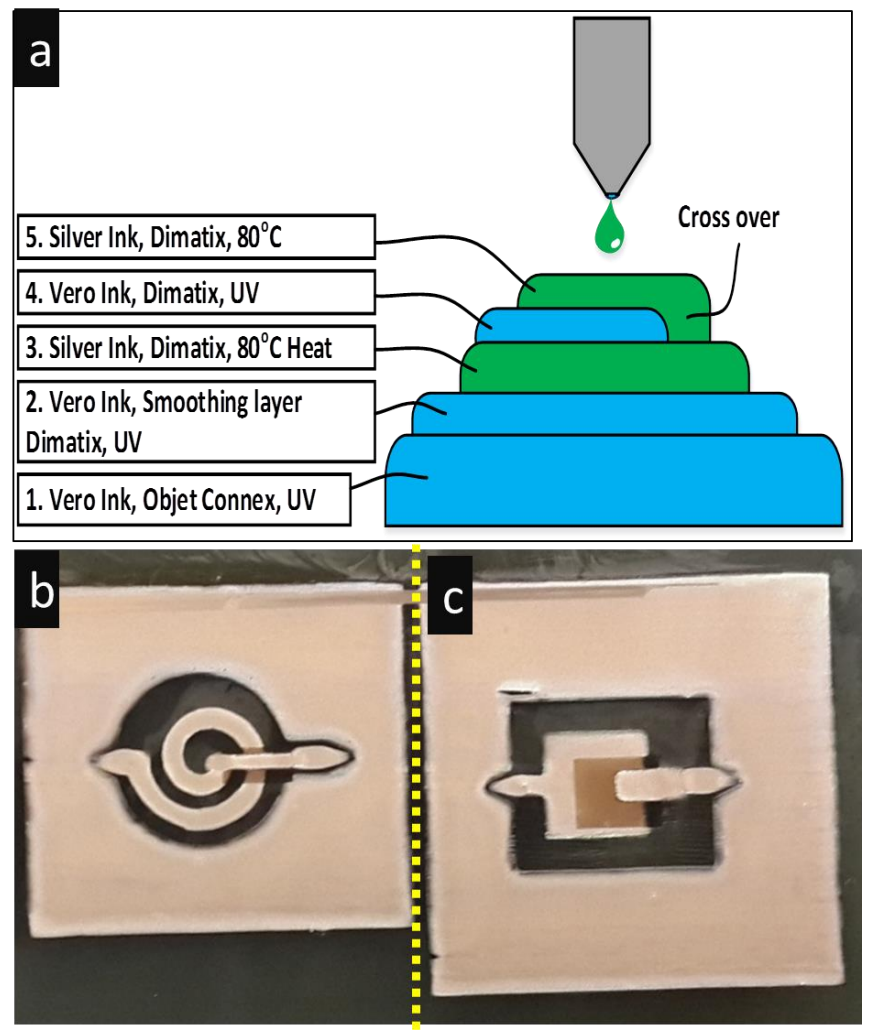

Fig. 2. (a) Depiction of printed multilayers stack up for inductors and capacitors, fabricated (b) inductor and (c) capacitor

\section{INK CHARACTERIZATION}

\section{A. 3D Printed UV Curable Vero Ink}

Stratasys UV cure ink is sold under the name "Vero", and comes in different colors and hardness. From the MSDS of hard Vero White, it is composed mostly of acrylic monomers $(<30 \%)$, isobornyl acrylate $(<25 \%)$, and various other components; acrylate oligomers and urethane acrylates. [10-12] Less than $1 \%$ titanium dioxide is used for the white color. The photoinitiator is Diphenyl-2,4,6 trimethylbenzoyl oxide $(<2 \%)$, which produces a free radical upon UV exposure, initiating the polymer reaction of the acrylic monomers and oligomers. The acrylic monomers are liquid at room temperature but too viscous for inkjet (125cp). Although the Vero ink is tailored for Stratasys Objet product line of 3D inkjet printers, it was more convenient to print with the Dimatix printer. The Dimatix allows control over nearly all print settings. By using a jetting temperature of $60{ }^{0} \mathrm{C}$ the viscosity of the Vero ink drops to $20 \mathrm{cp}$, allowing for excellent jetting from the Dimatix $10 \mathrm{pL}$ print head.

In order to design radio frequency components with the printable dielectric ink it is important to know the dielectric constant and dissipation factor. From the parallel plate measurements shown in Fig. 3a\&b, the Vero material has a dielectric constant of $\sim 3.0$ and a dissipation factor of $\sim 0.02$ up to $1 \mathrm{GHz}$.
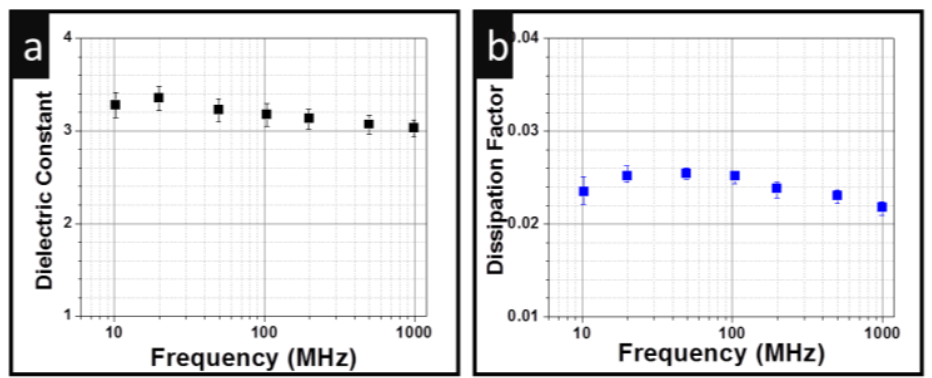

Fig.3. Dielectric properties of Stratysys Vero White UV cure acrylic polymer material. Bars represent the maximum and minimum measurement values of five test samples with the parallel plate method Agilent E4991 and dielectric test fixture 16453A

\section{B. Particle Free Silver-Organo-Complex (SOC) Based Metallic Ink}

An in house Silver-Organo-Complex (AOC) ink is utilized in this work which is stable and transparent with full details published elswhere. [13] The AOC ink provides an impressive $1 \times 10^{7} \mathrm{~S} / \mathrm{m},(20 \%)$ of bulk conductivity at only $80{ }^{0} \mathrm{C}$. Along with the high conductivity at low temperature the ink exhibits strong adhesion, long term stability (> 5months in a print head), and robust jetting performance. $3 \mathrm{D}$ printed material is tested with scotch tape without any removal of the silver film. Briefly, the ink is based on a silver-ethylamine-ethanolamineformate-complex based transparent and stable ink, wherein ethylamine, ethanolamine, and formate species act as in situ complexing solvents and reducing agents. As the ink is exposed with IR lamp for $5 \mathrm{~min}\left(\sim 80^{\circ} \mathrm{C}\right)$, elemental silver is the only phase that remains. Pure elemental silver results from the decomposition and reduction of complexes by the formate anion, which leads to the slow evaporation of ammonia and carbon oxide along with the low boiling point reactants. The as formulated ink was used with a drop-on-demand piezoelectric inkjet print head (Dimatix 16010) and a 2831 Dimatix printer. A standard waveform for the Dimatix model ink was and the voltage was varied for each nozzle to obtain $11 \mathrm{~m} / \mathrm{s}$ drop velocity ( 23V). 


\section{MEASUREMENT AND DISCUSSION}

Printed capacitors were measured with an Agilent 4980A LCR meter. Leakage current was measured with (Keithley 4200-SCS). High frequency measurements of the passive devices were taken in a two port configuration using $500 \mu \mathrm{m}$ pitch Z-probes and a cascade probe station with Agilent E8361A network analyzer.

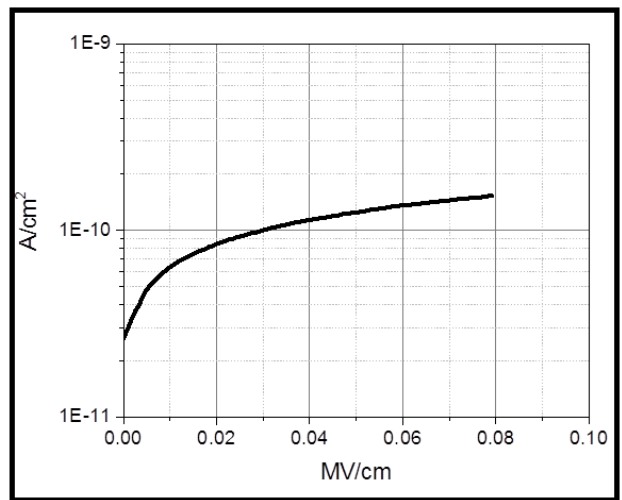

Fig. 4. Leakage current of a fully printed capacitor (tested to $100 \mathrm{~V}$ $\sim 0.08 \mathrm{MV} / \mathrm{cm}$ ) Dielectric thickness $12 \mu \mathrm{m}$.

\section{A. Breakdown of Capacitors}

Metal Insulator Metal (MIM) capacitors were printed with an in-house silver ink for electrodes (discussed in the above section). The capacitors have excellent leakage current values at around $1 \times 10^{-10} \mathrm{~A} / \mathrm{cm}^{2}$ at $0.08 \mathrm{MV} / \mathrm{cm}$ (equivalent to $100 \mathrm{~V}$ across the $12 \mu \mathrm{m}$ thick capacitor), as shown in Fig. 4.

\section{B. Low Frequency Measurement of Printed Metal Insulator Metal (MIM) Capacitors}

The capacitors were also tested against frequency and temperature, Fig. 5a\&b. While the dielectric behaves well at room temperature and up to $45{ }^{\circ} \mathrm{C}$, the material shows considerable dispersion at elevated measurement temperature. This is a known behavior in acrylic materials caused from dielectric relaxation at the glass transition temperature, $(\sim 50$ $\left.{ }^{0} \mathrm{C}\right)$. A thorough investigation of the dielectric relaxation in thin acrylic sheets has been studied by Wubbenhost et al describing this phenomenon.[14] A complete characterization of the
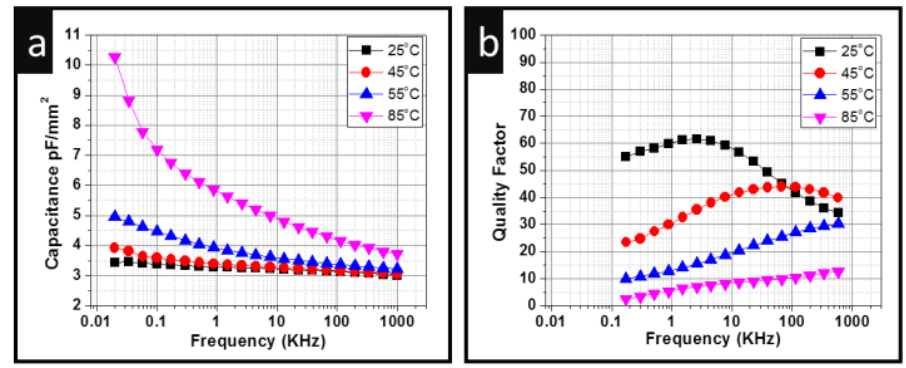

Fig. 5. (a) Capacitance versus frequency (b) quality factor, tested at $1 \mathrm{~V} \mathrm{AC}$ signal and 0 bias condition. $12 \mu \mathrm{m}$ thick printed layers. dielectric relaxation with temperature for this material is out of the scope of this work. The important point is that the dielectric is sensitive to temperature and should be operated below the glass transition. The Vero dielectric has also been tested against voltage bias and there is negligible change in capacitance value or quality factor. The major change in both physical and electrical dielectric properties occurs at elevated temperature. There is no issue so long as the material is processed below $80{ }^{\circ} \mathrm{C}$ to avoid excessive warping, and operated below $45{ }^{\circ} \mathrm{C}$ to avoid dielectric changes at the glass transition temperature.

\section{High Frequency Measurement for Printed Metal Insulator Metal (MIM) Capacitors and Inductors}

Fig. 6 is showing the performance of printed capacitor with different frequency. The capacitor is about $2 \mathrm{pF}$ and has a selfresonant frequency of $6.5 \mathrm{GHz}$. The quality factor of the capacitor starts out at 25 , and drops down to zero at selfresonance; this is consistent with low frequency measurements in Fig. 5.
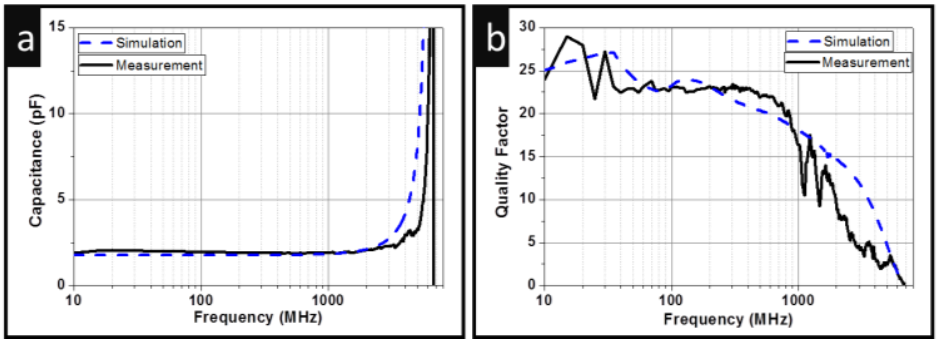

Fig.6. (a) Capacitor measured and simulated (b) quality factor (Capacitor area is $\sim 0.9 \mathrm{~mm} 2$ with a $12 \mu \mathrm{m}$ dielectric).

An inductor has also been tested as shown in Fig. 7a\&b. This 1.5 turn inductor has a self-resonance at $4 \mathrm{GHz}$, and is roughly $8 \mathrm{nH}$ at $1 \mathrm{GHz}$ frequency. The quality factor in simulation is slightly higher than the measurement at 7.5. This is likely due to more resistance from the printed ink than simulated, the inductors are extremely sensitive to conductivity and thickness of the printed ink. These inductors have been printed with 5 layers of SOC ink. The quality factors for both the inductors and capacitors are considered state-of-the-art among inkjet printed passives, even with the low $80{ }^{\circ} \mathrm{C}$ processing temperature. [7-8]
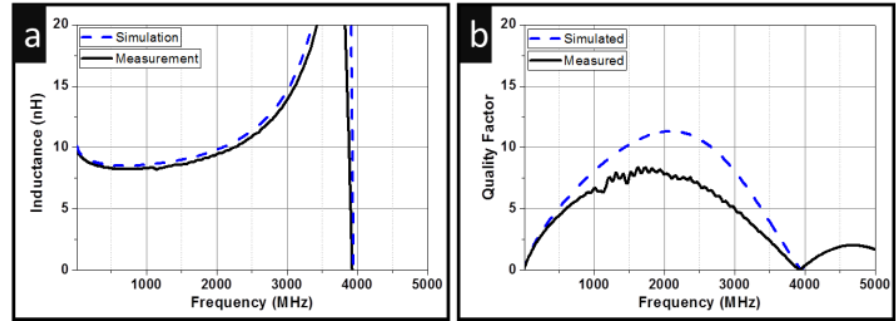

Fig.7. (a) Inductor measured and simulated (b) quality factor (1.5 turn inductor with an outer radius of $4 \mathrm{~mm}$, and $600 \mu \mathrm{m}$ thick lines). The inset in (a) is showing printed inductor. 


\section{CONCLUSION}

A process is presented where inkjet printed UV-cured polymer, and silver ink are integrated together. A major challenge is integrating a metal with the low temperature UV cure plastic material. A novel silver-organo-complex ink has been deployed, developed in-house which provides a conductivity of $1 \times 10^{7} \mathrm{~S} / \mathrm{m}$ at $80{ }^{0} \mathrm{C}$ in order to combine the materials effectively. An inductors and capacitors have been fabricated and measured to demonstrate the utility of the process. The capacitor and inductor exhibit state-of-the-art quality factors of $\sim 20$ and 8 respectively in the radio frequency regime. With future advances in printed metals, it is expected that performance could one day overcome conventional fabrication methods.

\section{ACKNOWLEDGMENT}

We acknowledge financial support from King Abdullah University of Science and Technology (KAUST) Office of Sponsored Research (OSR). For assistance and training with the objet printer, we greatly appreciate Rahman M. Hasan at the Visulation Facilities at KAUST.

\section{REFERENCES}

[1] J.-C. S. Chieh, B. Dick, S. Loui, and J. D. Rockway, "Development of a Ku-Band Corrugated Conical Horn Using 3-D Print Technology," IEEE Antennas Wirel. Propag. Lett, vol. 13, pp. 201-204, 2014.

[2] P. Nayeri, M. Liang, R. A. Sabory-Garcia, M. Tuo, F. Yang, M. Gehm, H. Xin, and A. Z. Elsherbeni, "3D Printed Dielectric Reflectarrays: Low-Cost High-Gain Antennas at Sub-Millimeter Waves," IEEE Trans. Antennas Propag., vol. 62 (4), pp. 2000-2008, 2014.
[3] C. Mariotti, M. M. Tentzeris, and L. Roselli, "Demonstration and Charaterization of Fully3D-printed RF Structures" Microwave Symposium (MMS), IEEE 15th Mediterranean, pp. 1-4, 2015.

[4] E. Geterud, P. Bergmark, and J. Yang, "Lightweight Waveguide and Antenna Components Using Plating on Plastics," In European Conference Antennas and Propagation; IEEE: Gothenburg, pp. 18121815,2013

[5] D. Redinger, S. Molesa, S. Yin, R. Farschi, and V.Subramanian, "An Ink-Jet-Deposited Passive Component Process for RFID,” IEEE Trans. Electron Devices, vol. 51, pp. 1978-1983, 2004.

[6] A. B. Menicanin, L. D. Zivanov, M. S. Damnjanovic, and A. M. Maric, "Low-Cost CPW Meander Inductors Utilizing Ink-Jet Printing on Flexible Substrate for High-Frequency Applications," IEEE Trans. Electron Devices, vol. 60, pp. 827-832, 2013.

[7] G. McKerricher, J. G. Perez, and A. Shamim, "Fully Inkjet Printed RF Inductors and Capacitors Using Polymer Dielectric and Silver Conductive Ink With Through Vias," IEEE Trans. Electron Devices, vol. 62 (3), pp. 1002-1009, 2015.

[8] B. S. Cook, C. Mariotti, J. R. Cooper, D. Revier, B. K. Tehrani, L. Aluigi, L. Roselli, and M. M. Tentzeris, "Inkjet-Printed, VerticallyIntegrated, High-Performance Inductors and Transformers on Flexible LCP Substrate," IEEE, pp. 1-4, 2014

[9] S.-Y. Wu, C. Yang, W. Hsu, and L. Lin, "3D-Printed Microelectronics for Integrated Circuitry and Passive Wireless Sensors," Microsyst. Nanoeng., vol. 1, pp. 15013, 2015.

[10] S. Magdassi, The Chemistry of Inkjet Inks, Ed., World Scientific: Singapore; Hackensack, NJ, 2010.

[11] Stratays. Material Saftey Data Sheet -Objet Vero White Plus RGD835. Stratasys July 2014.

[12] Stratasys. Polyjet Material Specifications - Vero White plus. Stratasys July 2014.

[13] M. Vaseem, G. McKerricher, and A. Shamim, "Robust Design of a Particle-Free Silver-Organo-Complex Ink with High Conductivity and Inkjet Stability for Flexible Electronics," ACS Appl. Mater. Interfaces, vol. 8 (1), pp. 177-186, 2016.

[14] M. Wubbenhorst, C. A. Murray, J. A. Forrest, and J. R. Dutcher, "Dielectric Relaxations in Ultra-Thin Films of PMMA: Assessing the Length Scale of Cooperativity in the Dynamic Glass Transition," In 11th International Symposium on Electrets, vol. ISE 11, pp 401-406, 2002. 